\title{
MANUFACTURING AND CONTINUOUS IMPROVEMENT AREAS USING PARTIAL LEAST SQUARE PATH MODELING WITH MULTIPLE REGRESSION COMPARISON
}

\author{
Carlos Monge ${ }^{1}$, Jesús Cruz Álvarez ${ }^{2}$, Jesús Fabián López ${ }^{3}$
}

\begin{abstract}
Structural equation modeling (SEM) has traditionally been deployed in areas of marketing, consumer satisfaction and preferences, human behavior, and recently in strategic planning. These areas are considered their niches; however, there is a remarkable tendency in empirical research studies that indicate a more diversified use of the technique.

This paper shows the application of structural equation modeling using partial least square (PLS-SEM), in areas of manufacturing, quality, continuous improvement, operational efficiency, and environmental responsibility in Mexico's medium and large manufacturing plants, while using a small sample $(n=40)$. The results obtained from the PLS-SEM model application mentioned, are highly positive, relevant, and statistically significant. Also shown in this paper, for purposes of validity, reliability, and statistical power confirmation of PLS-SEM, is a comparative analysis against multiple regression showing very similar results to those obtained by PLS-SEM. This fact validates the use of PLS-SEM in areas of untraditional scientific research, and suggests and invites the use of the technique in diversified fields of the scientific research.
\end{abstract}

JEL Classification Numbers: C12, C18, C39, DOI: http://dx.doi.org/10.12955/cbup.v2.442

Keywords: structural equations modeling, multiple partial least squares, PLS, SEM

\section{Introduction}

The structural equation modeling with latent variables (SEM), within the modalities covariance based (CB-SEM) (Bagozzi, 1994) and support of statistical software packages AMOS, EQS, LISREL and others or partial least squares based (PLS-SEM) (Reinartz, Haenlein, \& Henseler, 2009; Lohmöller, 1989) while using the SMART PLS software package, has been considered a quasi-standard statistical method for studies in the areas of management, market research, organizational behavior, management information systems, and consumer behavior (Hair, Ringle, \& Sarstedt, 2011; Hair, Sarstedt, \& Ringle, 2012; Henseler, Ringle, \& Sinkovics, 2009), as well as in advertising research. The desire to prove or check complete theories and concepts, as well as prediction (Hölck, Ringle, \& Sarstedt, 2010) is the reason for researchers to embrace this statistical technique (Hair et al., 2011; Henseler et al., 2009).

Unlike CB-SEM, PLS focuses on maximizing the explained variance of the endogenous latent variables instead of reproducing the theoretical covariance matrix (Henseler, Ringle, \& Sarstedt, 2011). In this regard, the decision to use CB-SEM and PLS-SEM is strongly dependent of the objective of the research study. If the goal is to prove or test theories, then CB-SEM would be a preferred method; whereas, if the objective is the development or prediction of theories, then it is advisable to use PLS-SEM (Hair et al., 2011, 2012; Henseler et al., 2011). In addition, PLS-SEM has some features that have promoted its popularity and can be highly beneficial when conducting research with small samples. Some of the features are that PLS-SEM is not restrictive in terms of sample size; in fact, it can work with small samples and does not assume normality of the input data, and can handle a formative scale. These advantageous features are not present in CB-SEM (Monge, Cruz, \& López, 2013; Hair et al., 2011, 2012; Henseler et al., 2011).

Despite the widespread popularity of SEM and particularly PLS-SEM in the social sciences, nearly 200 research studies were published in the top 20 marketing journals using PLS-SEM between 1980 and 2010 (Henseler et al., 2011). A few empirical studies have been conducted, using SEM applied to different areas such as manufacturing, quality, continuous improvement, sustainability and operational

\footnotetext{
${ }^{1}$ Carlos Monge Perry, Universidad Autónoma de Nuevo Léon (UANL), Mexico, carlosmongep@prodigy.net.mx

2 Jesús Cruz Álvarez, Universidad Autónoma de Nuevo León (UANL), Mexico, jesusphd@prodigy.net.mx

${ }^{3}$ Jesús Fabián López, Universidad Autónoma de Nuevo León (UANL), Mexico, fabian.lopezpz@gmail.com
} 
efficiency in manufacturing; some of these studies applied to these areas were made by Monge et al. (2013), Lee (2012), and Vinohd \& Dino (2012).

This article presents structural equation model using PLS-SEM, which interrelates lean manufacturing, sustainable manufacturing, continuous improvement in operational efficiency, and environmental responsibility in discrete manufacturing plants in Mexico. The study shows a positive and relevant impact as well as a statistically significant impact of the effectiveness of lean manufacturing (LME), sustainable manufacturing (SME) effectiveness, continuous improvement effectiveness (CIE) in operational efficiency, and environmental responsibility (OE\&ERI) in manufacturing plants of a heavily industrialized region of Mexico. It also shows the positive impact that the operational efficiency and environmental responsibility (OE\&ERI) have on the financial results (FR), carbon footprint impact (CFI), culture of sustainability of the organization (CSO), and employee satisfaction (ES) in Mexican manufacturing plants (Monge et al., 2013).

\section{Feasibility of PLS-SEM modeling in manufacturing, continuous improvement, and operational efficiency}

The PLS-SEM model presented in this paper was lacking a measure of good fit and a clear indicator of its statistical power, revealing the statistical representativeness of the sample and the predictive ability of endogenous constructs and their indicators thereof. Therefore, the model was constructed from exogenous constructs and their indicators instead, using in a way as a supplementary mathematical model based on multiple regression in order to validate the results of PLS-SEM model. It was further tested based on the scores of the latent constructs provided by the statistical package SMART PLS (Ringle, Wende, \& Will, 2005), and using these as independent variables, and operational efficiency and environmental responsibility as a dependent variable. This yielded values of $R, R^{2}$, adjusted $R^{2}$, the values of $p_{\text {value }}, \mathrm{t}, \mathrm{VIF}$, and $\mathrm{F}$, thus confirming the causal relationships, the explanatory power of the structural model, and the statistical power of the model, through Fisher's F and the absence of a significant effect of multicollinearity.

According to Hair et al., (2011, 2012), Henseler et al., (2011), and Monge et al., (2013), the previous exercise was conducted for verification purposes to complete the fulfillment of the diagnostic criteria to estimate the structural equation models PLS-SEM. The exercise was sufficient to conclude the validity of the measurement model and its scale, the predictive power of the structural model, and the causal relations and statistical power. It is noteworthy that the results issued by the SMART PLS package model discussed in this paper are very similar to those shown in the regression model run through the SPSS package, and thereby validated the PLS SEM model for use as a mechanism to predict future behavior in operational efficiency and environmental responsibility (OE\&ERI). In turn, the model can be used to predict the financial results (FR), carbon footprint impact (CFI), employee satisfaction (ES), culture of sustainability of the organization (CSO) in Mexican manufacturing plants (based on the data of the indicators of latent constructs), lean manufacturing effectiveness (LME), sustainable manufacturing effectiveness (SME), and continuous improvement (CIE).

\section{Operational efficiency and environmental responsibility (OE\&ERI) model operationalization, variables, and indicators}

The graphical representation of the operationalization of the model discussed in this article is presented in Figure 1. In this graph, both measurement models or external (outer) model and the structural or internal (inner) model are presented, as well as endogenous and exogenous latent variables, indicators, and the complex interrelationships that often accompany this type of structural equation models. It is noteworthy that a scale of reflective measurement included 34 items, consisting 
of a questionnaire conducted with key people in manufacturing plants. Items requested responses on Likert scale or hard data.

\section{Latent variables and their indicators}

Exogenous latent variables consist of Lean manufacturing effectiveness (LME). Endogenous latent variables consist of Sustainable manufacturing effectiveness (SME), continuous improvement effectiveness (CIE), and operational efficiency and environmental responsibility index (OE\&ERI). Exogenous indicators consist of lead time reduction (LTR), flow time reduction (FTR), and level of implementation of lean manufacturing (LILM). Endogenous indicators consist of Commitment to sustainability of the organization (OCS), environmentally friendly products production (EFPP), overcoming environmental regulations (OER), carbon footprint (CF), level of implementation of sustainable manufacturing (LISM), management commitment to continuous improvement (MCCI), employee involvement in continuous improvement (EICI), implanted suggestions (IS), level of implementation of the continuous improvement (LICI), carbon footprint impact (CFI), financial results (FR), employee satisfaction (ES), and culture of sustainability of the organization (CSO).

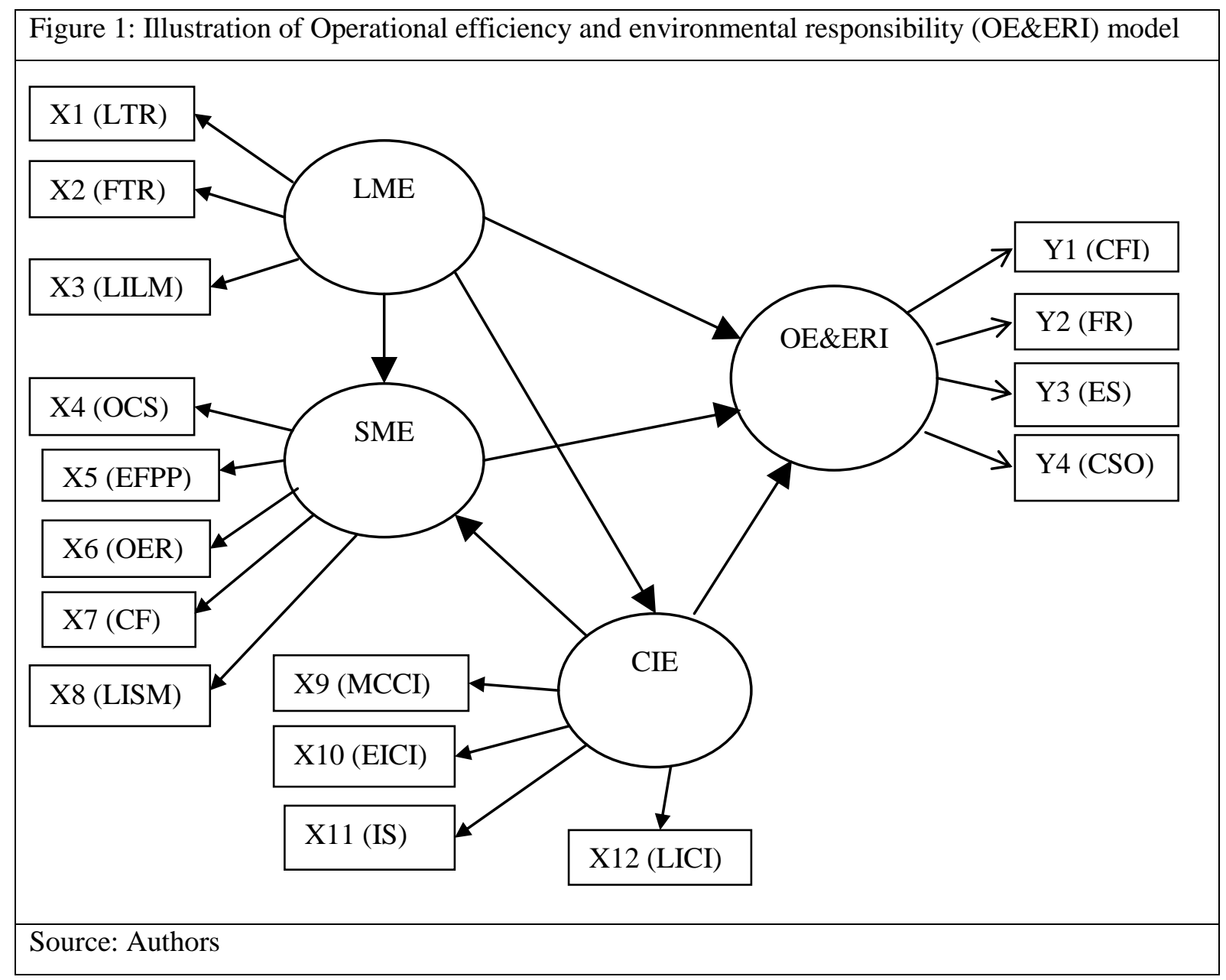

\section{Estimation of PLS-SEM and multiple regression models}

The model discussed in this paper was validated in compliance with all the quality criteria mentioned by Hair et al. (2011, 2012), Henseler et al. (2011), and Monge et al. (2013) for reflective scales. The model also shows that there is a positive, relevant and statistically significant impact of lean manufacturing, sustainable manufacturing, continuous improvement in operational efficiency, and environmental responsibility in manufacturing plants. 
This article presents the results of the estimation by PLS-SEM model, using a sample of 40 medium and large manufacturing plants, and data obtained through a survey applied to plant managers. The mix of the plants for its size is $72.5 \%$ (29) of large plants and $27.5 \%$ (11) of medium-sized plants. Their specialty is distributed as follows: metal-working at $32.5 \%$ (13), auto parts at $12.5 \%$ (5), electronic assemblies and electrical assemblies at $10.0 \%$ (4) each, thermoforming and ceramics at $7.5 \%$ (3) per specialty, conversion/paper and automotive assembly at $5.0 \%$ (2) each, aerospace and food and beverages at $2.5 \%$ each (1), and other manufacturing at $5.0 \%$ (2). Estimates of measurement models (outer model), structural (inner model), as well as the supplementary multiple regression models are described in the following sections.

\section{Estimation and results of the measurement model (outer model) of PLS-SEM}

The estimation of the measurement model was performed using SMART PLS 2.0 (Ringle et al., 2005), using the PLS algorithm for $\mathrm{n}=40$, and the quality criteria established by Hair et al. $(2011,2012)$, Henseler et al. (2009), and Monge et al. (2013). The results of outer model estimation are presented in Figure 2. The assessment of PLS-SEM models, with reflective scales and multiple items, must be submitted for confirmation for their validity and reliability, as well as the predictive ability of the model to predict behavior of endogenous constructs SME, CIE, and OE\&ERI from the constructed exogenous SME (Coelho \& Henseler, 2012; Hair et al., 2011; Monge et al., 2013).

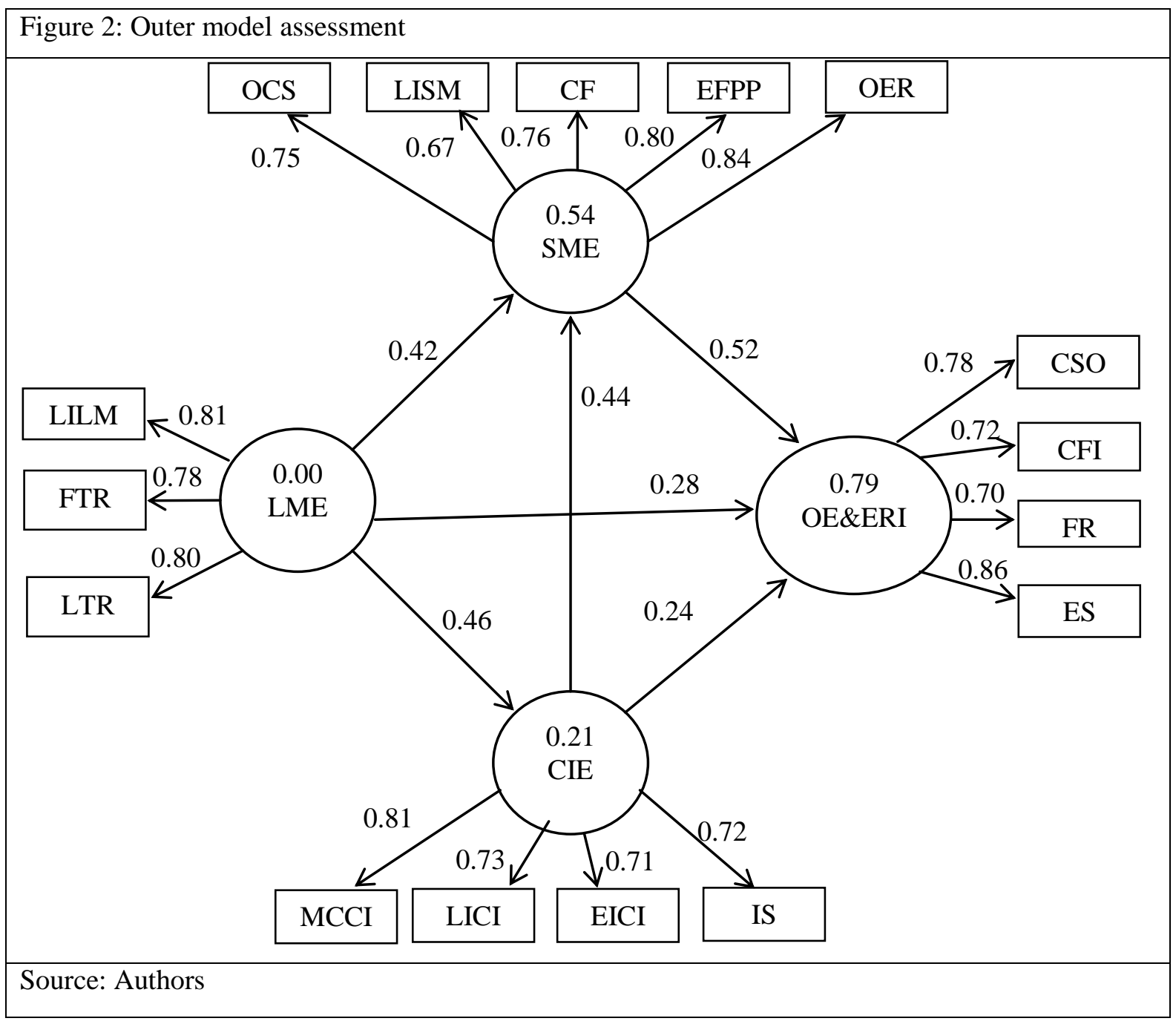

In Figure 2, the circled values are the coefficients of determination; $R^{2}$ values of $0.25,0.50$ and 0.75 represent explanatory power of the latent variables as low, medium, and significant, respectively. Quantities shown on the arrows of the measurement model (external) are standardized loads; however, 
it is important to distinguish between reflective and formative models when evaluating measurement. Reflective models should be evaluated based on the reliability and validity of the constructs; for these purposes, the composite reliability ( $\rho c)$ is used as an estimate of the internal consistency and of the construct according to Hair et al. (2011) and Henseler et al. (2009). The measured values must be greater than 0.70 (exploratory research on the values of 0.60 to 0.70 are acceptable). Table 1 shows that the values of the composite reliability $(\rho c)$ for LME, SME, CIE, and OE\&ERI constructs exceed 0.80, indicating internal consistency in the constructs of the model discussed in this work of Monge et al. (2013). The results, not shown in Figure 2, of the run of the PLS, bootstrapping, and blindfolding algorithms with SMART PLS 2.0 (Ringle et al., 2005) are presented in Table 1, which are required to complete the assessment and analysis of the results.

Table 1: Average variance extracted (AVE), composite reliability, and Cronbach's alpha values

\begin{tabular}{|c|r|r|r|}
\hline Construct & AVE & Composite reliability (pc) & \multicolumn{1}{|c|}{ Cronbach's alpha } \\
\hline CIE & 0.55 & 0.83 & 0.73 \\
\hline LME & 0.64 & 0.84 & 0.75 \\
\hline SME & 0.59 & 0.88 & 0.82 \\
\hline OE\&ERI & 0.59 & 0.85 & 0.77 \\
\hline
\end{tabular}

Source: Authors

The indicators of the measurement model must also show reliability, to validate the fact that their standardized loads, according to Hair et al. (2011) and Henseler et al. (2009), should have values greater than 0.70. In Figure 2, it is shown that most of the loads of the indicators exceed this value, so it is possible to imply that the reliability and validity of the measurement instrument are valid. In the case of standardized load corresponding to 0.67 LIMS indicator, this indicator was not removed because, according to (Hair et al., 2011), only those indicators with standardized loadings lower than 0.70 must be excluded, if their elimination raises reliability composite ( $\rho c$ ) over the critical threshold - this was not the case.

The assessment of validity of reflective measurement model focuses on the convergent validity and discriminant validity. For convergent validity, the values of the average variance extracted (AVE) should be greater 0.50 for all constructs, as shown in Table 1. These values exceed the level mentioned, indicating a sufficient degree of convergent validity, which means that a specific latent variable explains more than half of the variance in comparison to their corresponding indicators (Hair et al., 2011).

\section{Assessment and results of the structural or internal model (inner model) of PLS-SEM}

Internal model estimation was performed by using SMART PLS 2.0 (Ringle et al., 2005) and the bootstrapping algorithm, with 40 cases of the same quantity of manufacturing plants. The results are shown in Figure 3. The quantities shown on the arrows, external measurement model and structural model (internal), represent the values of the Student $\mathrm{T}$ test of interrelations within latent variables and test of latent variables and their indicators, which indicates that the level of significance of cause-effect relations are at a level not less than $95 \%$.

It is important to remember that structural equation modeling using partial least square (PLS-SEM) does not assume that data of the cases are normally distributed. Thus, it uses nonparametric-applying PLS bootstrapping procedure, which means that it performs repeated random sampling with 
replacement from the original sample to create a bootstrap sample, and, with it, obtains standard errors for hypothesis testing. The process assumes that the distribution of the samples is a reasonable representation of the population distribution. The bootstrap sample allows the estimated coefficients by PLS-SEM algorithm to be tested for their level of significance (Hair et al., 2011; Henseler et al., 2009).

The primary criteria for evaluating the structural model are the measurements of the $\mathrm{R}^{2}$ and the significance level of the path coefficients. Considering that the objective of PLS-SEM is to predict or explain the variance of the endogenous variables, it is necessary that $\mathrm{R}^{2}$ values of the key constructs are high, or set high depending on the discipline of the research in question. $\mathrm{R}^{2}$ values of $0.75,0.5$ or 0.25 for endogenous latent variables in the structural model are considered substantial, moderate, and weak respectively (Hair et al., 2011).

Referring back to Figure 2, $\mathrm{R}^{2}$ values range from weak, in the case of the CIE, to moderate and substantial, in the latent variables SME and OE\&ERI. The structural model shows, in particular, the value of $R^{2}$ of the endogenous latent variable, OE\&ERI, which has a substantial value of 0.79 (shown in Figure 2). These values were obtained by using the bootstrap algorithm in SMART PLS 2.0 with 5000 samples (Ringle et al., 2005).

To assess the level of significance of the path coefficients, the bootstrap algorithm was used. According to Hair et al. (2011) and Henseler et al. (2009), critical values of T are 1.65 for a two-tailed test $($ significance level $=0.10$ or $10 \%$ ), 1.96 (significance level $=0.05$ or $5 \%$ ), and 2.58 (significance level $=0.01$ or $1 \%$ ). Figure 3 shows the values of $\mathrm{T}$ for the structural model. Except for the value of $\mathrm{T}$ for CIE $\rightarrow$ OE\&ERI, of 2.26, which is slightly more significant than 0.05 or $5 \%$, the rest of the values is significant by at least 0.01 or $1 \%$ with respect to the values of $\mathrm{T}$ measurement model (external); these are significant by at least 0.01 or $1 \%$.

Regarding the predictive relevance, the blindfolding algorithm of the SMART PLS 2.0 (Ringle et al., 2005) was run. One of the objectives and contributions, that this study pursues, is that the research model (Model OE\&ERI) has a predictive capacity of the behavior of the endogenous construct (OE\& ERI), so that the model may guide users of the model in their improvement efforts in running the model and obtaining values for the latent variable OE\&ERI. Furthermore, in regards to the assessment of the ability to predict or predictive relevance of the structural model, blindfolding algorithm PLSSEM was used to obtain the cross-validated redundancy (CV Red). The predominant measurement is $\mathrm{Q}^{2}$, which establishes that the structural model must be able to accurately predict each indicator of endogenous latent construct (Hair et al., 2011); in this study, the latent variable is OE\&ERI.

Table 2: Cross validated redundancy CV Red $\left(\mathrm{Q}^{2}\right)$ values

\begin{tabular}{|c|c|r|}
\hline Construct & 1-SSE/SSO CV Red Q \\
\cline { 2 - 3 } & CIE & 0.55 \\
\cline { 2 - 3 } & LME & 0.64 \\
\cline { 2 - 3 } & SME & 0.59 \\
\cline { 2 - 3 } & OE\&ERI & 0.59 \\
\hline
\end{tabular}

Source: Authors

$\mathrm{Q}^{2}$ is obtained by using the blindfolding algorithm, a sample reuse technique that omits each piece of data, and uses the resulting estimate to predict the missing part. The blindfolding procedure applies only to endogenous latent constructs with reflective measurement model. In order to make the estimation of $\mathrm{Q}^{2}$, the CV Red measurement of a particular endogenous latent construct must have values greater than 0.00 , which is interpreted as a predictive or explanatory relevance of latent exogenous constructs - for the endogenous constructs under consideration. In the case of this article, 
it can be seen from Table 2 that the values of CV Red for the constructs have values greater than 0.00 , confirming the predictive relevance of the exogenous constructs on endogenous constructs. In other words, it is possible to predict the behavior of endogenous constructs, SME, CIE, and OE\&ERI in this case, from the exogenous constructs.

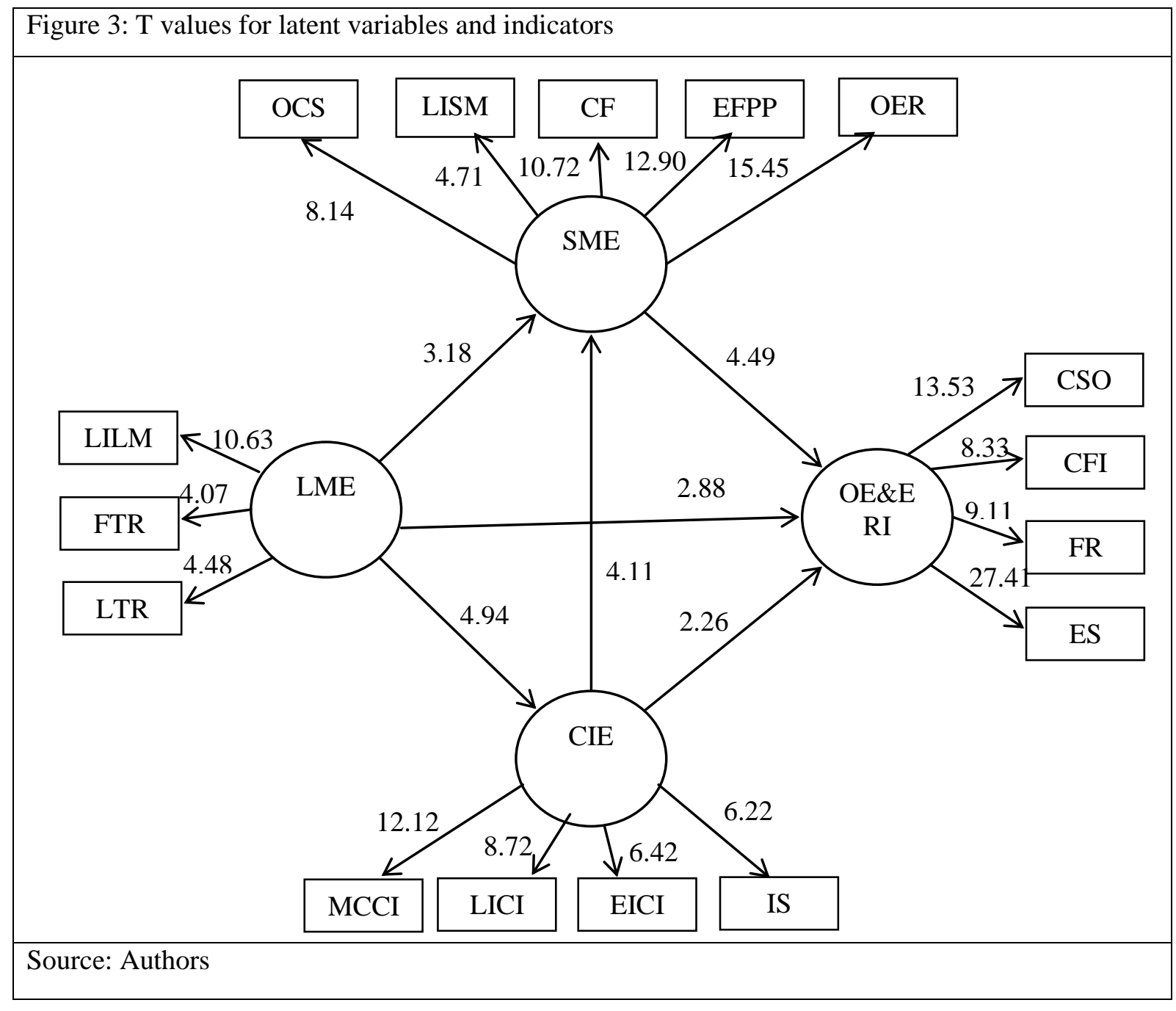

\section{Estimation of supplementary multiple regression models}

Henseler et al. (2009) and Hölck et al. (2010) reported that because SEM-PLS does not have a mean for measuring the goodness of fit that exists in the recent literature, supplementary studies that complement PLS-SEM based researches were used instead. The additional studies use multivariate analysis techniques, such as multiple regression and some other techniques. In this article, we have decided to include supplementary studies of multiple linear regression with two purposes: (1) to test the statistical power of the model PLS-SEM using the Fisher F that cannot be obtained directly from the software PLS SMART 2.0 (Ringle et al., 2005), and (2) to develop a model to predict the behavior of OE\&ERI, from statistically significant indicators of latent variables LME, CIE, SME and OE\&ERI, which will further enable a third purpose - to predict the latent construct OE\&ERI using the unstandardized scores of relevant indicators for each construct.

\section{Development of the model to determine the statistical power of OE\&ERI}

To develop the supplementary model, multiple linear regression was used by inputting the standardized scores (Henseler et al., 2011), obtained directly from the run of the software SMART PLS 2.0 (Ringle et al., 2005), of the latent variables LME, CIE, SME and OE\&ERI; OE\&ERI is the 
dependent variable, while the rest of the aforementioned are independent variables. The resulting model for $n=40$, and the results are shown in Figure 4 and Table 3.

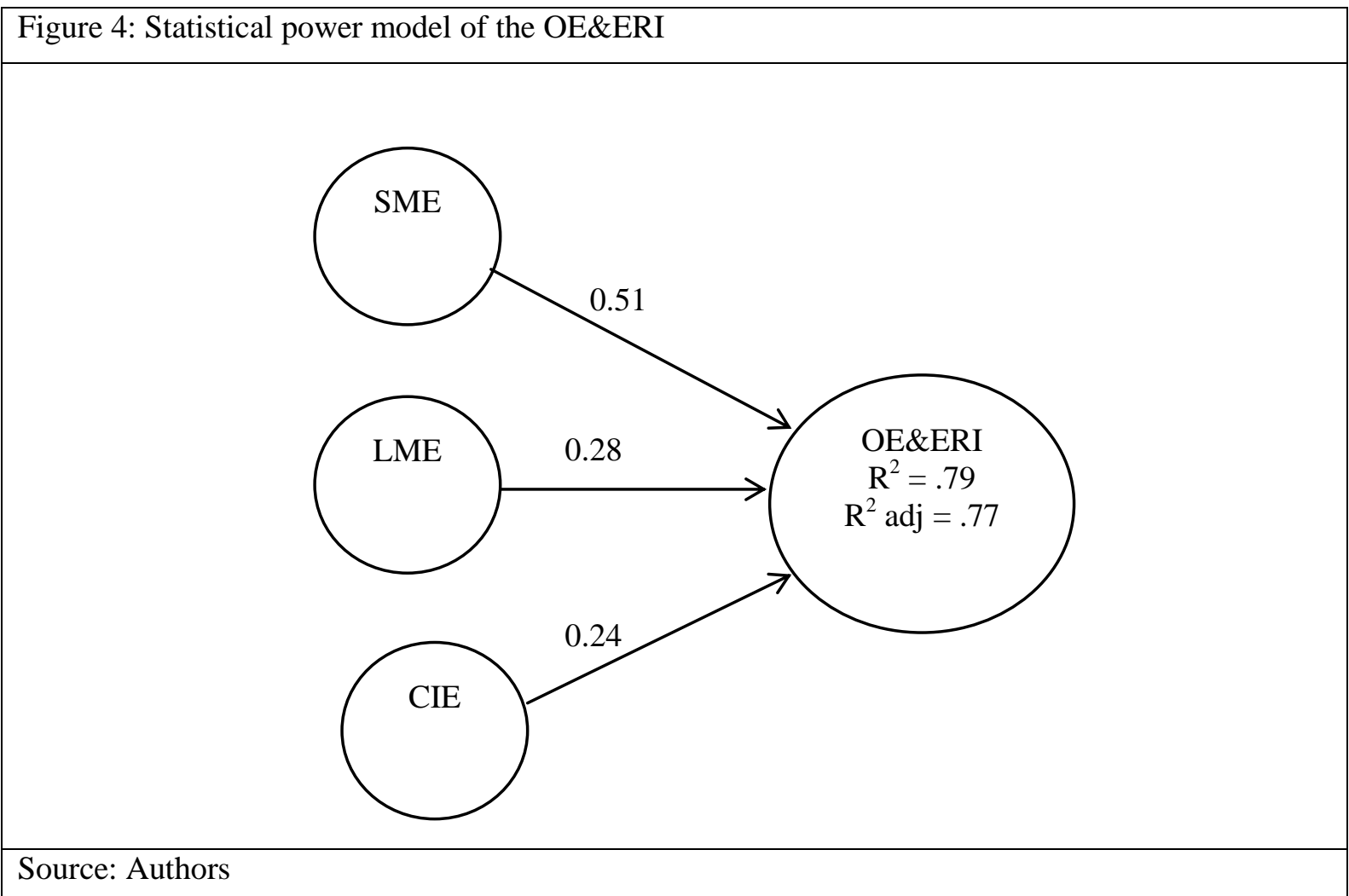

When the regression model using SPSS is run, a value of $\mathrm{R}^{2}$ of 0.79 is obtained for OE\&ERI; this result is very similar to that obtained by the structural equation model using PLS-SEM. Standardized betas have similar trends to those of PLS-SEM. Regarding the quality values to assess, the regression models (such as FIV, $\mathrm{t}$ and $\mathrm{p}$, and $\mathrm{F}$ ) are shown below in Table 3.

It can be seen that $t$ and $p$ values are significant at least in 0.05 (5\%), and the FIV value is less than 5, which shows a non-significant collinearity effect (Hölck et al., 2010). The statistical power of the model denoted by $\mathrm{F}$ is well above the threshold value of 3.49, which fulfills the first purpose of testing the statistical power of the PLS-SEM model proposed and discussed in this article.

\section{Development of predictive model of OE\&ERI}

In developing the predictive model OE\&ERI, the unstandardized scores of the indicators of the variables LME, SME, and CIE were used; the results were statistically significant after running a regression model using SPSS and "stepwise" method considering all indicators. Once the stepwise method showed the relevant indicators, they were fed into a multiple regression model. The results are shown in Figure 5.

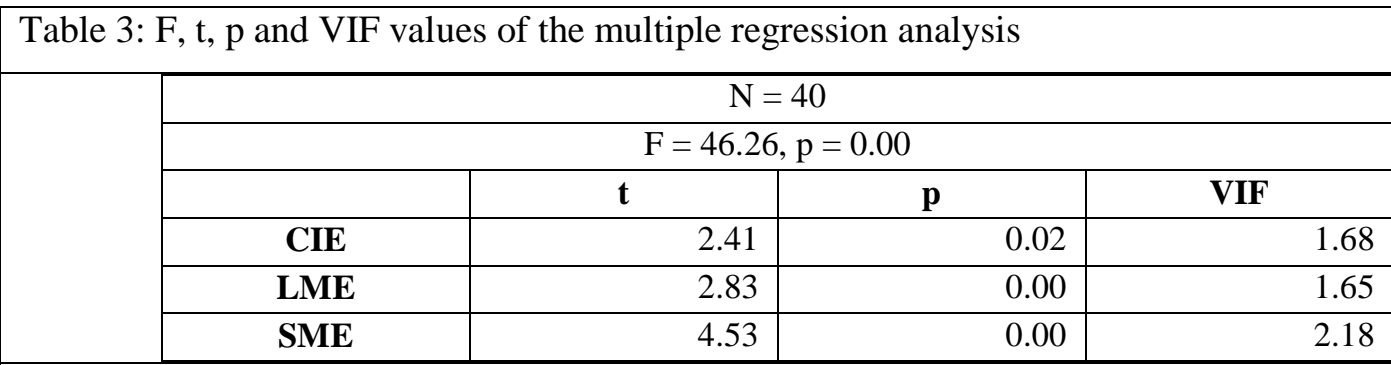

Source: Authors 
In Figure 5 , it can be seen that an $\mathrm{R}^{2}$ value is 0.83 , which is considered significant; and since the values on the arrows are standardized betas, it is possible to determine which indicator has the most and the least impact on OE\&ERI. In this case, LIMS and LILM have the most and least impact, respectively, which may suggest that it is crucial to work on items using LIMS as an indicator. In other words, analyzing the items that feed the LILM, FTR, IS, LIMS, and EFPP indicators can extract prescriptive actions to guide improvement actions. The OE\&ERI model, showing the values of the unstandardized betas, are shown in Figure 6 and Table 4. From these values, it is possible to extract the regression equation for predicting effects. The values shown in Table 4, Figures 5 and 6 enable us to determine the predictive equations of the OE\&ERI model discussed in this study. These equations are shown in the following sections of this paper, both for the cases of the usage of standardized and unstandardized betas, along with corresponding equations.

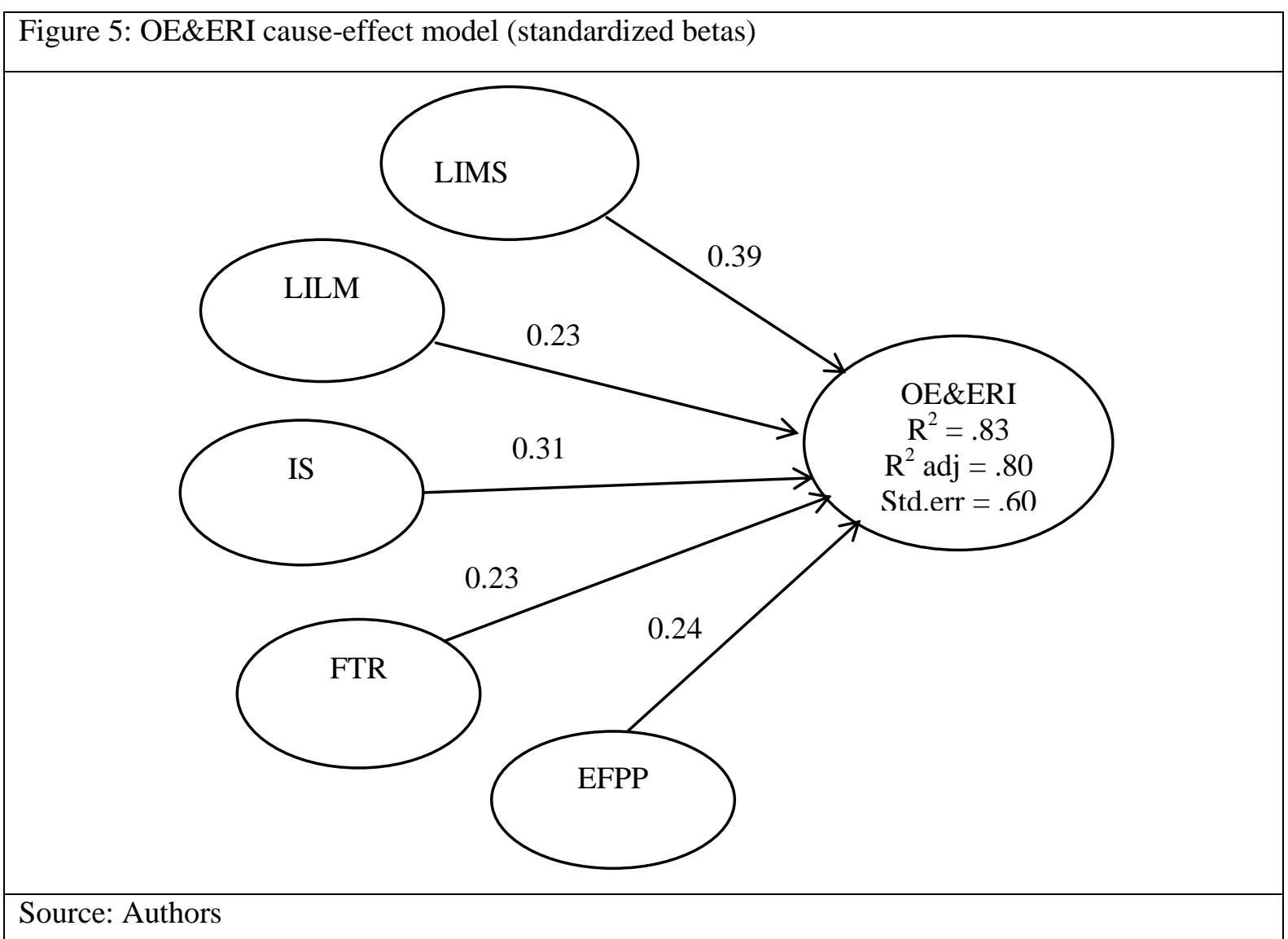

Table 4: F, t, p \& VIF values of the OE\&ERI model

\begin{tabular}{|l|r|r|r|r|r|r|}
\hline & \multicolumn{1}{|c|}{ Unst. betas } & \multicolumn{1}{c|}{ Std. err } & \multicolumn{1}{c|}{ Std. betas } & \multicolumn{1}{c|}{ t } & \multicolumn{1}{c|}{ p value } & \multicolumn{1}{c|}{ FIV } \\
\hline Constant & 0.34 & 0.26 & - & 1.29 & 0.21 & 1.49 \\
\hline LIMS & 0.08 & 0.02 & 0.39 & 4.52 & 0.00 & 1.69 \\
\hline LILM & 0.01 & 0.00 & 0.22 & 2.44 & 0.02 & 1.08 \\
\hline IS & 0.01 & 0.00 & 0.31 & 4.17 & 0.00 & 1.16 \\
\hline FTR & 0.02 & 0.00 & 0.23 & 2.97 & 0.00 & 1.79 \\
\hline EFPP & 0.26 & 0.11 & 0.24 & 2.49 & 0.02 & \\
\hline
\end{tabular}

Source: Authors 
$\mathrm{F}, \mathrm{t}, \mathrm{p} \&$ VIF values the resulting regression equations, corresponding to OE\&ERI cause-effect model, are listed in Table 4.

The regression equation takes the following form:

$$
O E \& E R I=0.34+0.08 * L I M S+0.01 * L I L M+0.01 * I S+0.02 * F T R+0.26 * E F P P
$$

The value of $F$ is 32.81, with $p=0.00$, and a value of the Durbin-Watson test of 1.57. The values of $t$, $\mathrm{p}$ and FIV are shown in Table 4, and are seen to be significant in at least 0.05 (5\%). Table 4 further reveals that there is no significant multicollinearity between the independent variables of regression in the present FIV values because they are below the level of 5 (Hölck et al., 2010).

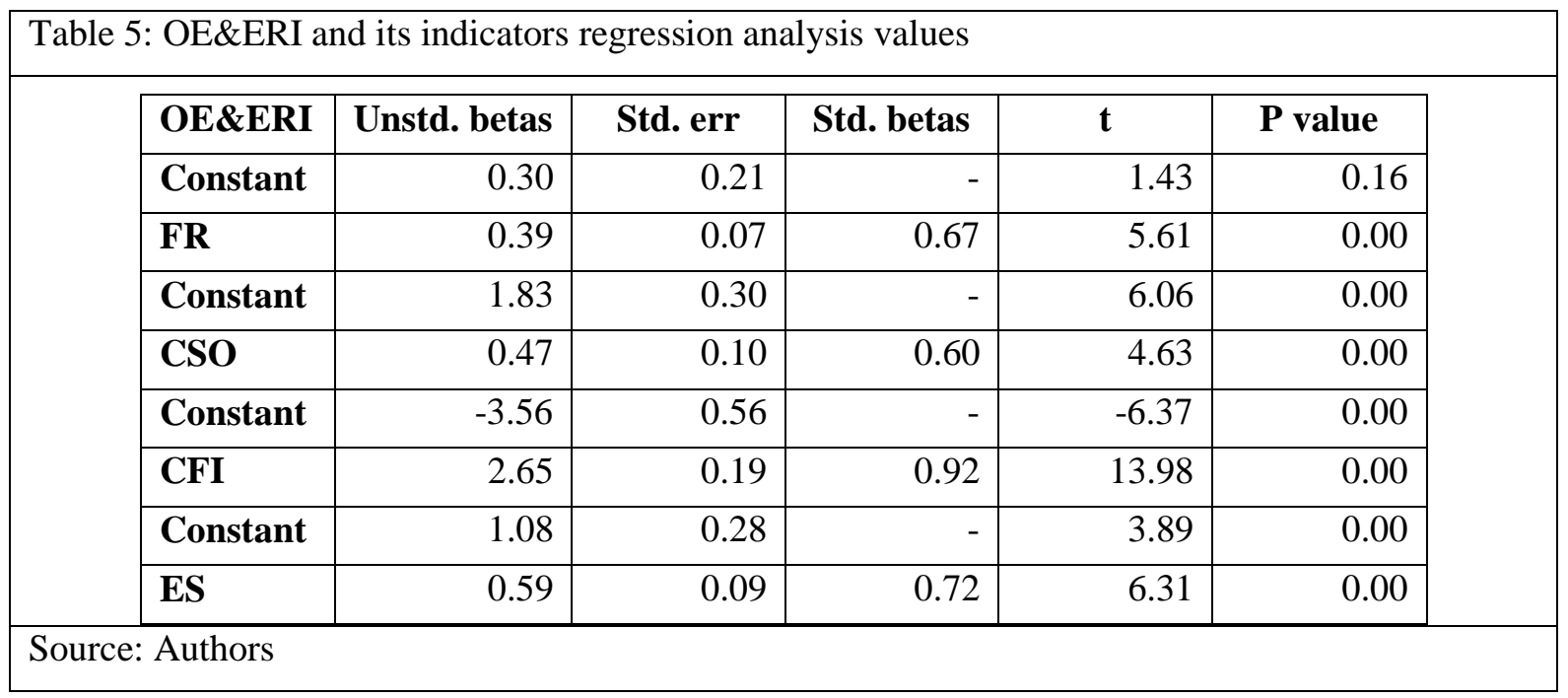

Figure 6: OE\&ERI predictive model (unstandardized betas)

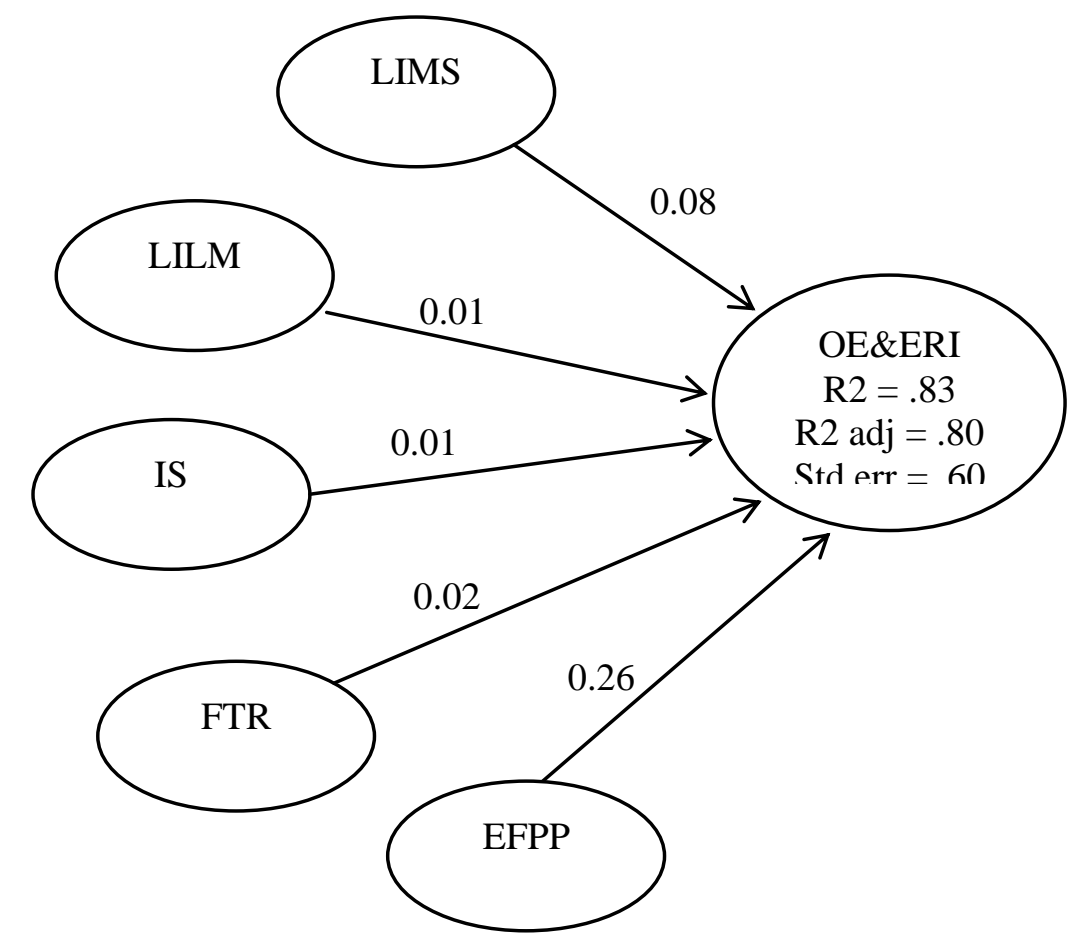

Source: Authors 


\section{Predictive model of the indicators of OE\&ERI}

The indicators of OE\&ERI can be estimated from the model shown in Figure 7, while using the original unstandardized scores of the OE\&ERI and a simple linear regression using SPSS statistical software. It can also be seen in the results shown in Figure 7 that the OE\&ERI model explains a higher percentage indicator behavior CFI (0.84) and less to the indicator CSO (0.36). The regression equations and the criteria ( $\mathrm{t}, \mathrm{p})$ are shown in equations 2 through 5 and in Table 5 , respectively. Prediction equations of OE\& ERI indicators are:

$$
\begin{gathered}
F R=0.30+0.29 * O E \& E R I, \mathrm{~F}=31.48, \mathrm{p}=0.00, \mathrm{DW}=2.00 \\
C S O=1.83+0.47 * O E \& E R I, \mathrm{~F}=21.47, \mathrm{p}=0.00, \mathrm{DW}=2.30 \\
C F I=-3.56+2.65 * O E \& E R I, \mathrm{~F}=195.38, \mathrm{p}=0.00, \mathrm{DW}=2.59 \\
E S=1.08+0.59 * O E \& E R I, \mathrm{~F}=39.88, \mathrm{p}=0.00, \mathrm{DW}=2.35
\end{gathered}
$$

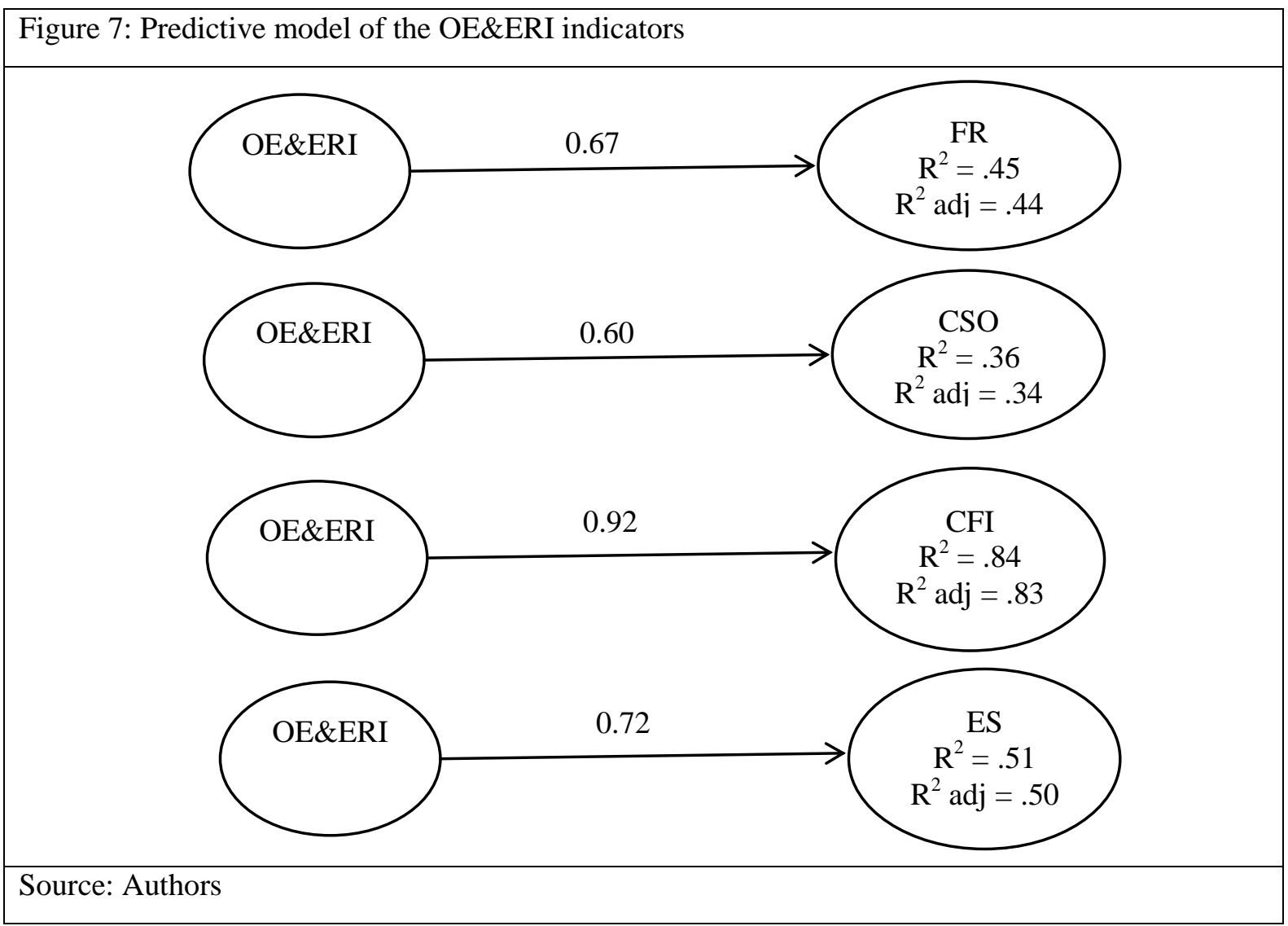

\section{Discussion}

As mentioned throughout this article, the structural equation models CBS-SEM (Bagozzi, 1994) or PLS-SEM (Lohmöller, 1989), have been widely and increasingly used in social science-related research and consumer behavior study. However, SEM can also be used in an empirical research in engineering areas such as: manufacturing, quality, continuous improvement, and operational efficiency of organizations (Monge et al., 2013; Lee, 2012; Vinohd \& Dino, 2012). The use of SEM in research in these areas can generate positive, relevant, and statistically significant results, such as the empirical study presented in this article, which deals with the study of cause-effect relationships of lean manufacturing, sustainable manufacturing, continuous improvement on operational efficiency, and environmental responsibility in the case of medium-sized and large discrete manufacturing plants of Mexico. 
The empirical study discussed in this article returns reliable and valid results, which are also statistically significant, and shows predictive ability of the model to estimate future performance of manufacturing plants in operational efficiency and environmental responsibility. These results were also retested to demonstrate validity and reliability in their significance and statistical power. For these purposes the following criteria were used: (1) the quality criteria for PLS-SEM models, recommended by Hair et al. $(2011,2012)$ and Henseler et al. (2011); and (2) supplementary study of a multiple regression model according to Henseler et al. (2011, 2009). The application of both techniques showed similar results, which may suggest that SEM can be used reliably in research either to verify and or to develop theories (Hair et al., 2011). It is also applicable for predictive purposes (Monge et al., 2013; Hair et al., 2011; Hölck et al., 2010), in different areas aside from those traditionally addressed by structural equation approaches.

\section{Conclusion}

The structural equation models CB-SEM and PLS-SEM can be used, in a reliable and statistically significant manner, to test theories (CB-SEM), to develop theories, or to predict endogenous constructs behavior (PLS-SEM). The models can also be utilized in other arenas outside of marketing, consumer behavior, advertising, and psychology. In the particular case of PLS-SEM, this technique described in this article, can be used in the areas of manufacturing, quality and continuous improvement, etc. It may also be accompanied by supplementary statistical models, such as regression to estimate performance in the operational efficiency of key manufacturing facilities indicators, which enable organizations to make decisions and to prioritize specific actions for improvement with an empirical basis (Hölck et al., 2010).

\section{References}

Bagozzi, R., (1994). Structural equation models in marketing research: Basic principles. In P. Richard, \& P. Bagozzi (Eds.), Principles of Marketing Research. Oxford, UK: Blackwell.

Coelho, P., \& Henseler, J. (2012). Creating customer loyalty through service customization. European Journal of Marketing, 46(3), 331-356.

Hair, J., Ringle, C. \& Sarstedt, M. (2011). PLS-SEM: Indeed a silver bullet. Journal of Marketing Theory and Practice, 19(2), 139-151.

Hair, J., Sarstedt, M., \& Ringle, C. (2012). An assessment of the use of partial least squares structural equation modeling in marketing research. Journal of the Academy of Marketing Science, 40, 414-433.

Henseler, J., Ringle, C., \& Sarstedt, M. (2011). Using partial least squares path modeling in advertising research: Basic concepts and recent issues. In Handbook of research on international advertising, Northampton, USA: Edward Elgar.

Henseler, J., Ringle, C., \& Sinkovics, R. (2009). The use of partial least squares path modeling in international marketing. Advances in International Marketing, 20, 277-319.

Hölck, C, Ringle, C., \& Sarstedt, M. (2010). Management of multi-purpose stadiums: Importance and performance measurement of services interfaces. International Journal Services Technology and Management, 14(2/3), 188-204.

Lee, S. (2012). The impact of manufacturing practices on operational performance. Review of Business Research, 12(5), 184189.

Lohmöller, J. (1989). Latent variable path modeling with partial least squares. Heidelberg: Physica.

Monge, C., Cruz, J., \& López, F. (2013). Impacto de la manufactura esbelta, manufactura sustentable y mejora continua en la eficiencia operacional y responsabilidad ambiental en México. Información Tecnológica, 24(4), 15-31.

Reinartz, W., Haenlein, M., \& Henseler, J. (2009). An empirical comparison of efficacy of covariance-based and variancebased SEM. International Journal of Research in Marketing, 26, 332-344.

Ringle, C., Wende, S., \& Will, A. (2005). Smart PLS 2.0M3; Next generation path modeling software. Retrieved from: http://www.smartpls.de

Vinohd, S., \& Dino, J. (2012). Structural equation modeling of lean manufacturing practices. International Journal of Production Research, 50(6), 1598-1607. 